\title{
Verletzungen des Tracheobronchialbaums
}

\author{
Injuries to the Tracheo-bronchial Tree
}

Autoren

Institute
S. Welter ${ }^{1}$, H. Hoffmann ${ }^{2}$

${ }^{1}$ Thoraxchirurgie und thorakale Endoskopie, Ruhrlandklinik am Universitätsklinikum Essen, Essen, Deutschland

${ }^{2}$ Chirurgie, Thoraxklinik am Universitätsklinikum, Heidelberg, Deutschland

\section{Schlüsselwörter}

- Notfalleingriff

- Penetrationsverletzung

- Stenose

- Thoraxchirurgie

Key words

emergency intervention

- penetrating injuries

- stenosis

- thoracic surgery

\section{Bibliografie}

DOI http://dx.doi.org/

10.1055/s-0032-1328269

Zentralbl Chir 2013; 138:

111-116 @ Georg Thieme

Verlag KG Stuttgart · New York .

ISSN 0044-409X

\section{Korrespondenzadresse}

Dr. Stefan Welter, MD

Ruhrlandklinik

Thoraxchirurgie und thorakale

Endoskopie

Tüschener Weg 40

45239 Essen

Deutschland

Tel.: $0201 / 4334012$

Fax: 0201/4334019

stefan.welter@

ruhrlandklinik.uk-essen.de

\section{Zusammenfassung}

$\nabla$

Verletzungen des Tracheobronchialbaums sind seltene, aber meist lebensbedrohliche Ereignisse, deren adäquates Management eine hohe thoraxchirurgische Expertise erfordert. Die Genese der Verletzung - iatrogen oder traumatisch - gibt erste wichtige Hinweise auf das Verletzungsmuster. Die Bronchoskopie ist die zentrale Diagnostik zur Abschätzung des Ausmaßes der Verletzung. Typische iatrogene Verletzungen der Tracheahinterwand werden je nach Schweregrad konservativ oder operativ behandelt. Im Gegensatz dazu erfordern traumatische stumpfe oder penetrierende Verletzungen des Tracheobronchialbaums regelhaft das chirurgische Vorgehen, welches typischerweise eine technisch anspruchsvolle Rekonstruktion des Atemwegs beinhaltet.

\section{Einleitung}

Verletzungen der Trachea und der Hauptbronchien sind selten, aber meist lebensbedrohlich. Sie sind so selten, dass auch in großen Zentren in Deutschland in der Regel weniger als 5 Patienten pro Jahr gesehen werden [1-4]. Sie sind andererseits meist so akut lebensbedrohlich, dass sehr schnell die Weichen für ein adäquates Management gestellt werden müssen. Die folgende Übersicht soll aus der Sicht des Praktikers dem versorgenden Chirurgen Anhaltspunkte für zielorientiertes Handeln liefern.

Erster wichtiger Anhaltspunkt ist die Ursache der Verletzung: Iatrogene oder traumatische (stumpfe bzw. penetrierende) Verletzung? Da in Deutschland 3 von 5 Verletzungen des Tracheobronchialbaums iatrogenen Ursprungs sind, d.h. als Folge medizinischer Maßnahmen auftreten [1], soll diese Verletzung vorrangig erläutert werden.

\section{Abstract \\ $\nabla$}

Tracheobronchial injuries are rare events but often life-threatening. A great expertise in the field of thoracic surgery is necessary to manage these events. The aetiology of the injury - traumatic or iatrogenic - allows immediate considerations about the pattern of damage. The flexible or rigid bronchoscopy is the central diagnostic procedure to classify the injury. Whereas iatrogenic tracheal lacerations can be treated by conservative or operative means depending on severity, traumatic injuries of the tracheo-bronchial system need technically challenging operative reconstructions in almost every case.

\section{latrogene Verletzungen des Tracheobronchialbaums}

Immer dann, wenn ohne vorangegangenen Lungeneingriff Blut aus dem Tubus abzusaugen ist oder unter Beatmung sich rasch ein deutliches Weichteilemphysem entwickelt, muss an eine iatrogene Verletzung des Tracheobronchialbaums gedacht werden.

Iatrogene Tracheaverletzungen sind typischerweise Lazerationen der Tracheahinterwand, überwiegend im mittleren und unteren Drittel, in der Medianlinie oder rechts lateral davon, gelegentlich bis in den rechten Hauptbronchus reichend. $\mathrm{Zu}$ solchen Verletzungen kommt es selten bei elektiver Intubation, häufiger bei Notfallintubationen und im Rahmen dilatativer Tracheostomie-Anlagen [1]. Die hohe Letalität iatrogener Tracheaverletzungen von 10\% [5] bis zu $42 \%$ in manchen berichteten Serien $[6,7]$ ist allerdings meist in der Schwere der Begleiterkrankungen begründet. So ist die ursächliche Notfallintuba- 
tion oft im Rahmen einer Reanimation oder schwerer akuter respiratorischer Störungen bei Pneumonie, Lungenembolie oder Myokardinfarkt erfolgt.

\section{Mögliche Pathomechanismen}

Ursächlich werden eine übermäßige Gewaltanwendung im Rahmen der Notfallintubation, das zu späte Zurückziehen des überstehenden Führungsstabs oder die Intubation mit starren Tracheoskopen genannt. Ein weiterer, oft diskutierter Mechanismus ist die Tubusbewegung bei geblocktem Cuff [8]. Untersuchungen an Kadaver-Tracheen zeigten jedoch, dass ein Tubus-Cuff erst bei einer Füllung mit $70 \mathrm{ml}$ Luft gegen erheblichen Widerstand zur Hinterwandruptur führt, sodass dieser Mechanismus als eher unwahrscheinlich angesehen werden kann [9]. Während der Dilatationstracheotomie gibt es zahlreiche Fehlermöglichkeiten, die eine Hinterwandverletzung bei der Einführung des Bougie begünstigen können: Fehlende bronchoskopische Kontrolle, Fehlpunktion von lateral in die Trachea, Punktion durch einen Trachealknorpel mit entsprechendem Widerstand beim Einführen des Bougie und der Tracheostomiekanüle. $\mathrm{Zu}$ den technischen Fehlern kommt fast immer noch ein patienteneigener Risikofaktor dazu: Viele iatrogene Verletzungen der Tracheahinterwand betreffen übergewichtige und kleine Frauen im Alter zwischen 50 und 70 Jahren. Ein kurzer Hals, laufende Kortikoidtherapie und die Verwendung von Doppellumentuben werden als weitere Risikofaktoren genannt $[1,10]$.

\section{Klinik}

Die typischen klinischen Zeichen sind Blut im Tubus oder Bluthusten nach Extubation, thorakales Weichteilemphysem und Luftnot als Folge eines Pneumothoraxes oder der Schwellung des Larynxes durch die Fortleitung des Mediastinalemphysems. Beatmungsprobleme ergeben sich durch ein hohes Leckage-Volumen entlang des Cuffs oder über eine einliegende Thoraxdrainage und durch Verrutschen der Tubusspitze in das Mediastinum mit plötzlichem Anstieg des Beatmungswiderstands. Die notwendige Erhöhung des Beatmungsdrucks verschlimmert das Mediastinalemphysem erheblich und kann rasch zum Auftreten eines Spannungspneumothoraxes führen.

\section{Diagnostik}

Ziel der Diagnostik ist die schnelle Abschätzung der Tragweite der Verletzung, d.h. die Bestimmung von Länge und Tiefenausdehnung der Verletzung, Eingrenzung des betroffenen Tracheaabschnitts und Beurteilung des Ausmaßes der Komplikationen: Hautemphysem und Pneumothorax.

Erste Hinweise zur Beurteilung von Hautemphysem, Pneumothorax und Tubuslage ergibt die Röntgenübersichtsaufnahme des Thoraxes. Als unmittelbare Konsequenz kann direkt die Anlage einer Thoraxdrainage notwendig sein. Wegweisend ist die Bronchoskopie. Dabei sollte ein flexibles Bronchoskop mit ausreichend kaliberstarkem Saug- und Spülkanal zum Einsatz kommen. Das Bronchoskop wird vorsichtig in die Trachea eingeführt, Blut und Fibrin abgesaugt und zunächst die Hauptkarina identifiziert. Danach wird das Bronchoskop und - beim beatmeten Patienten - der Tubus langsam bis an den kranialen Verletzungsrand zurückgezogen und das Ausmaß der Verletzung, die Hernierung des mediastinalen Gewebes in das Tracheallumen und die Blutungsaktivität bestimmt. Bei transmuralen Verletzungen der Pars membranacea muss zudem immer eine Ösophagusverletzung ausgeschlossen werden.
Tab. 1 Schweregradeinteilung der iatrogenen Tracheaverletzung nach Cardillo [11].

Level I: Oberflächliche Verletzung von Mukosa oder Submukosa Level II: Verletzung der Muscularis ohne Mediastinalemphysem Level III A: Komplette Lazeration der Hinterwand mit Weichteilhernierung oder Mediastinalemphysem

Level III B: Lazeration der Trachealwand mit Ösophagusverletzung

oder Mediastinitis

Kürzlich wurde eine Klassifikation der Tiefenausdehnung (Level I-III b) von Cardillo et al. [11] vorgeschlagen ( Tab. 1). Verletzungen des Levels II betreffen die gesamte Muscularis und die der Level III sind transmurale Verletzungen mit Mediastinalemphysem und Weichteilhernierung. Abschließend wird - bei weiterbestehender Beatmungspflichtigkeit - der Tubus distal der Verletzung platziert und mit so geringem Druck wie möglich geblockt. Ist der Patient kardiorespiratorisch ausreichend stabil, sollte die Diagnose mittels Spiral-CT des Thoraxes verifiziert werden und Begleitverletzungen wie Hämatome ausgeschlossen werden. Pathognomonisch ist dabei die mediastinale Luftansammlung um die Trachea herum sowie die auffällige Erweiterung des Tracheallumens im Bereich des Cuffs [12,13]. Selten können die rupturierten Lippen der Hinterwand separat identifiziert werden. Vorzugsweise erfolgt diese Diagnostik in einer erfahrenen thoraxchirurgischen Abteilung, in der falls erforderlich direkt die operative Versorgung durchgeführt werden kann.

\section{Akutmanagement}

Als Akutmaßnahmen sind Beruhigung und antitussive Medikation des wachen Patienten notwendig. Ggf. ist eine bronchoskopische Absaugung von Blutkoageln und Sekret erforderlich, um den Hustenreiz zu minimieren. Beim intubierten Patienten sollte die Tubusspitze distal der Läsion platziert und der Cuff nur mit dem minimal notwendigen Druck geblockt werden. Bei Vorliegen eines Pneumothoraxes besteht die Indikation zur Einlage einer Thoraxdrainage. Eine ca. $1 \mathrm{~cm}$ lange Inzision der Haut unterhalb der Claviculae kann sehr schnell eine erhebliche Entlastung von Haut- und Mediastinalemphysem erbringen mit Erleichterung der Atemarbeit. Jeder Nachweis eines Kreislaufeinbruchs als Hinweis auf einen Spannungspneumothorax sollte in dieser Situation zur umgehenden Notfallanlage mindestens einer rechtsseitigen, besser beidseitiger Thoraxdrainagen führen.

\section{Definitive Therapie der iatrogenen \\ Tracheahinterwandverletzung}

A. Am einfachsten und meist erfolgreich ist die konservative Therapie [2]. Ob dies möglich ist, entscheidet sich meist unmittelbar. Spontan atmende Patienten mit einer Level-I-II- und einige mit einer Level-III A-Verletzung erfordern in der Regel keine operative Revision; gleiches gilt für beatmete Patienten, wenn sich der Tubus sicher distal der Verletzung platzieren lässt.

Die konservative Therapie umfasst die Überwachung und Beruhigung des wachen Patienten oder die mehrtägige Beatmung des Patienten mit Begleiterkrankung, bis eine ausreichend stabile Fibrinschicht die Läsion überdeckt $[4,8]$. Vereinzelt beschrieben ist auch die bronchoskopische Fibrinauftragung insbesondere bei nicht transmuralen Läsionen [11]. Diese Behandlung ist hinsichtlich der Mortalität risikoarm und wird daher von einigen Autoren favorisiert. Unter einer Breitspektrum-Antibiotikatherapie lässt sich darüber hinaus die früher so gefürchtete Mediastinitis [14] fast immer vermeiden. Allerdings zeigt eine Fallserie von Conti 


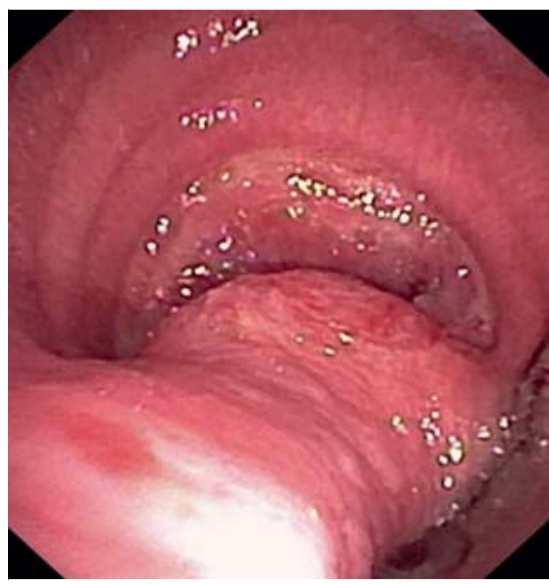

Abb. 1 Bronchoskopische Diagnostik wegen „Trachealstenose" 8 Wochen nach konservativ behandelter Tracheahinterwandverletzung. Aufnahme während des Hustens. Normalkalibrige Trachea unter CPAP-Beatmung. Subtotaler Kollaps beim Husten.

et al. [8] zahlreiche mit der konservativen Behandlung assoziierte Probleme wie z.B. Notwendigkeit der nicht invasiven Beatmung, mehrtätige einseitige Ventilation, Notwendigkeit zur operativen Ausräumung mediastinaler Serome, Auftreten einer tracheoösophagealen Fistel, verzögerte Heilung $>4$ Wochen etc. Aus der eigenen Ambulanz ist ein Fall bekannt, der 8 Wochen nach Tracheahinterwand-Verletzung einen exspiratorischen Totalkollaps der Trachea beim Husten durch Protrusion mediastinalen Gewebes entwickelte ( $\bullet$ Abb. 1). Weiterhin scheint bei adipösen Patienten initial die Mediastinalprotrusion erheblich zur Luftnot und respiratorischen Erschöpfung beizutragen ( $\square$ Abb.2). Hier spiegelt sich die Tatsache wider, dass die konservative Behandlung zwar zu einer stabilen Verklebung beider Wundlefzen mit dem dahinterliegenden mediastinalen Gewebe führt, nicht jedoch zu einer Adaptation der Wundränder miteinander. Über mittel- und langfristige funktionelle Aspekte der verschiedenen Therapien existieren bisher keine Untersuchungen.

B. Operative Versorgung: Eine absolute Operationsindikation sind immer Begleitverletzungen des Ösophagus, entsprechend einer Verletzung Level IIIb nach Cardillo [4,11], und meistens jede größere Kommunikation der offenen Trachea mit der Thoraxhöhle [4]. Zunehmendes Hautemphysem, schwergradige respiratorische Insuffizienz mit Notwendigkeit der Intubation, Schwierigkeiten mit der mechanischen Ventilation, Pneumothorax mit Luftfistel über die liegende Drainage sowie Mediastinitis sind relative Indikationen für ein chirurgisches Vorgehen [2]. Durch die operative Nahtversorgung wird die Verletzung idealerweise anatomiegerecht verschlossen, eine Luftleckage sicher beendet und damit das Pneumothoraxrisiko verringert, einer Mediastinitis vorgebeugt und die iatrogen verursachte Verletzung zielgerichtet behandelt. Operative Zugangswege für ein offenchirurgisches Vorgehen sind die posterolaterale Thorakotomie rechts [15] oder die kollare Inzision mit der Möglichkeit zur Erweiterung durch partielle oder vollständige Sternotomie [10,16]. Darüber hinaus sind videoassistierte Vorgehensweisen von kollar aus beschrieben $[17,18]$.

Nachteil der Thorakotomie ist das zusätzliche signifikante Operationstrauma bei den schon kritisch kranken Patienten. Bei jedem transzervikalen Zugang muss zudem die Trachea eröffnet oder quer durchtrennt werden, bevor die Hinterwand erreicht werden kann $[17,18]$. Einen Ausweg aus diesem Dilemma könnte hier die endotracheale Nahtversorgung über ein $14 \mathrm{CH}$ starres Tracheoskop bieten, die keinen operativen Zugangsweg benötigt ( Abb. 3) [19]. In der Essener Arbeitsgruppe haben wir inzwischen Erfahrung mit 10 Patienten. Alle hatten eine transmurale Hinterwandverletzung mit ausgeprägtem Mediastinalemphysem und waren intubiert und beatmet. Drei weitere Fälle konnten nicht endoluminal versorgt werden, da der Patient keine Jet-Ventilation tolerierte oder die Ruptur unmittelbar am Übergang zwischen Knorpel und Pars membranacea lag und keine seitliche Membranlefze vorhanden war, um die Nadel einzustechen. Die OP-Zeit ist inzwischen von 115 min auf minimal 40 min zurückgegangen. Bisher wurden keine operationsassoziierten Komplikationen wie Rekurrensparese, Ösophagusverletzung oder Mediastinitis beobachtet. Ein Patient ist innerhalb von $48 \mathrm{~h}$ an einem erneuten Myokardinfarkt verstorben. Ein respiratorisch stabiler Patient kann danach am OP-Tag extubiert werden.

Der Einsatz tracheobronchialer Stents zur Versorgung trachealer Verletzungen ist bisher nur in Form von Fallberichten publiziert [20]. Nach über 15-jähriger dokumentierter Erfahrungen in Heidelberg $[5,21]$ und in Essen und unter Berücksichtigung der Erfahrungen mit dem Einsatz von Stents bei malignen Erkrankungen (Sekretverlegung, Stent-Migration, Granulationsgewebestenose, ...) sehen wir bei typischen Verletzungen der Tracheahinterwand keine Indikation für den Einsatz endotrachealer Stents; allenfalls bei einem komplexen Verletzungsmuster und fehlenden Alternativen mag dies eine Option sein.

\section{Traumatische Verletzungen des Tracheobronchialbaums \\ $\nabla$}

Stumpfe oder penetrierende Verletzungen der Atemwege im Rahmen stumpfer Traumata sind oft schon am Unfallort letal. Die Prognose der Patienten, die trotzdem das Krankenhaus erreichen, hängt im Wesentlichen von der Sicherung der Atemwege und dem Ausmaß der Begleitverletzungen ab. Von allen Patienten, die mit einem Thoraxtrauma das Krankenhaus erreichen, haben 1-2\% eine tracheobronchiale Verletzung. Stumpfe Thoraxtraumata mit Verletzungen der intrathorakalen Atemwege sind regelhaft mit anderen Begleitverletzungen assoziiert [22,24,25].

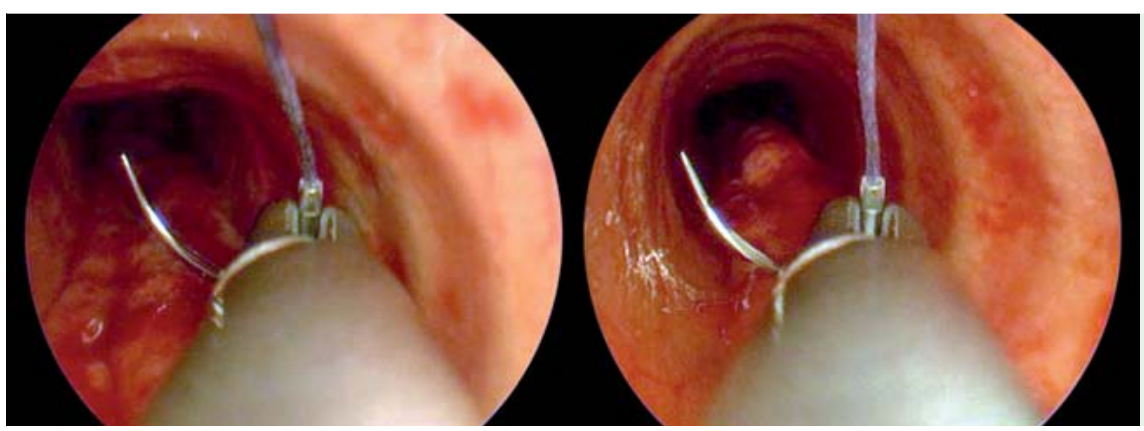

Abb. 2 Schwer adipöse Patientin mit einer Intubationsverletzung der Tracheahinterwand im Rahmen eines kleinen Routineeingriffs am Kniegelenk. Respiratorische Erschöpfung $1 \mathrm{Std}$. nach Extubation. Endobronchial Nachweis einer massiven exspiratorischen Fettgewebsprotrusion bei Husten und Pressen. Therapie: Endotracheale Naht, Extubation am Folgetag, Entlassung nach einer Woche. Jet-Ventilation, links inspiratorische Phase, rechts exspiratorische Phase. 


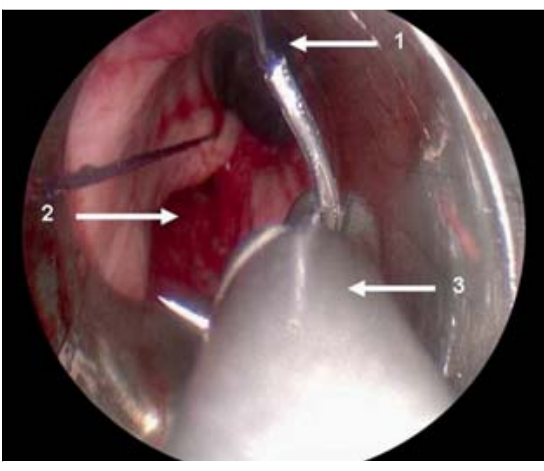

Abb. 3 Fortlaufende endotracheale Naht einer Tracheahinterwandverletzung unter Jet-Ventilation. 1 = Sicht auf die distale Trachea, 2 = Ruptur der Trachea hinterwand, 3 = optischer Nadelhalter.

Bis zu 75\% aller traumaassoziierten Todesfälle sind auf Thoraxtraumen zurückzuführen [26]. An tödlich verletzten Unfallopfern mit Thoraxtrauma wird eine Rate von 2,8\% tracheobronchialer Verletzungen angegeben [10,27]. Penetrierende Verletzungen der Atemwege betreffen zu 75\% die zervikale Trachea. Bei penetrierenden Thoraxverletzungen kommt es nur bei ca. $1 \%$ zu einer Mitbeteiligung der Trachea.

\section{Pathophysiologie der Verletzung}

Stumpfe Verletzungen treten bei Verkehrsunfällen, Sturz aus großer Höhe und bei Verschüttung auf. In den meisten Fällen resultiert eine transversale Ruptur (74\%), die überwiegend die intrathorakalen Luftwege innerhalb eines Radius von $2,5 \mathrm{~cm}$ um die Bifurkation betrifft (96\%) [28-30]. Dabei rupturiert am ehesten die Membran zwischen den Knorpelspangen. Drei Mechanismen können das Verletzungsmuster erklären [9,22,31]:

1. Ein massiver Druckanstieg in den Atemwegen während des stumpfen Traumas bei geschlossener Glottis führt zur Ruptur an einer Prädilektionsstelle.

2. Durch Kompression des Sternums Richtung Wirbelsäule werden die Lungen nach lateral gedrängt und Scherkräfte führen zu Rupturen im Bereich der Bifurkation mit typischerweise Quetschung bzw. Abriss des rechten Hauptbronchus.

3. Eine starke Dezeleration (Schleudertrauma, Gurtfixierung) führt zum Einriss oder Abriss der Trachea gegenüber eher fixierten Strukturen wie dem Krikoid oder der Bifurkation.

Bei penetrierenden Verletzungen durch Messerstiche, Pfählung im Rahmen von Arbeitsunfällen oder als Schussverletzungen können die zervikale, die thorakale Trachea oder die Hauptbronchien betroffen sein. Penetrierende Thoraxtraumata sind selten auf den Tracheobronchialbaum beschränkt [23]. So wurde in einer Arbeit von Inci et al. [32] bei 755 Patienten mit penetrierender Verletzung des Thoraxes in 190 Fällen ein Hämatothorax, in 184 Fällen ein Hämatopneumothorax, in 144 Fällen ein Pneumothorax sowie Zwerchfellrupturen und andere komplexe Verletzungen bei $<150$ Patienten beschrieben. Auch stumpfe Thoraxtraumen gehen gehäuft mit schweren Begleitverletzungen einher $[33,34]$.

Schussverletzungen sind kritisch, da der Eintrittskanal keine Auskunft darüber gibt, welche Organe durch das Projektil verletzt wurden, insbesondere wenn kein korrespondierender Austrittskanal gefunden und das Geschoss im Körper verblieben ist. Verletzungen durch Schusswaffen sind in der Regel komplex, da durch Anprall an festere Strukturen wie z.B. Wirbelkörper eine Richtungsänderung des Projektils auftreten kann. Hochgeschwindigkeitsgeschosse hinterlassen ein weit größeres Schadensausmaß als initial erkennbar. Die kinetische Energie des Geschosses wird beim Gewebedurchtritt teilweise abgegeben. Die
Ausdehnung des zerplatzenden Gewebes verursacht kurzzeitig eine Höhlenbildung. Obwohl sich das Gewebe dann wieder zusammenzieht, ist das Gewebetrauma immer weit größer, als Einund Austrittskanal vermuten lassen [22-24].

Klinik

Tachypnoe (59\%) und Hautemphysem (81\%) sind die häufigsten Hinweise auf eine tracheobronchiale Verletzung [33]. Weiterhin können leichte Hämoptysen sowie Dysphonie, Stridor und Zyanose auftreten. Eine kollabierte Lunge, die trotz regelrecht einliegender Pleuradrainage nicht zur Ausdehnung kommt, ist ein typischer Hinweis auf eine zentrale Atemwegsverletzung oder eine massive Parenchymverletzung [25,28]. Pathognomonisch für eine Atemwegsverletzung ist der Luftaustritt aus einer offenen Halsverletzung.

\section{Diagnostik}

Die Röntgenübersichtsaufnahme des Thoraxes ist bei ca. $88 \%$ aller tracheobronchialen Verletzungen auffällig [33]. Pneumothorax, Haut- und Mediastinalemphysem sind typisch bei intrathorakalen Verletzungen und seltener bei zervikalen Tracheaverletzungen. Ein zervikales Hautemphysem ist auf einer lateralen Halsaufnahme zuverlässiger zu sehen als in der Thoraxübersicht [9]. Ein kompletter Bronchusabriss zeigt sich in einem fehlenden Hilus und einer Komplettatelektase der Lunge [12]. Jeder stabile Patient mit vermuteter Atemwegsverletzung sollte eine ThoraxCT und eine Bronchoskopie, ggf. Ösophagoskopie erhalten [35]. Die Identifizierung einer Ösophagusverletzung ist prognostisch von besonderer Bedeutung, da eine verzögerte Behandlung nach $24 \mathrm{~h}$ mit einer steigenden Letalität verbunden ist [35]. In der Thorax-CT ist eine Tracheaverletzung immer mit einem Mediastinalemphysem und/oder tief-zervikalem Emphysem assoziiert (100\%): Weitere Zeichen sind: Paratracheale Luft (93\%), Pneumothorax (36\%), retroperitoneale Luft (14\%), Konturunterbrechung der Trachealwand (71\%), Trachealring-Fraktur (14\%), Tubus-CuffDistention oder -Hernierung (71\%). Insgesamt kann bei $85-100 \%$ aller zervikalen oder thorakalen Trachealverletzungen die Diagnose mittels CT erhärtet werden $[9,13]$. Allerdings kommen alle genannten radiologischen Zeichen auch bei Lungentraumatisierten ohne Atemwegsverletzung vor, daher muss die Verdachtsdiagnose immer durch Bronchoskopie überprüft werden. Durch die flexible oder starre Bronchoskopie können Blutkoagel abgesaugt, eine Blutungsquelle identifiziert, die Verletzung selbst beurteilt oder eine Lumenverlegung im Verletzungsbereich dargestellt werden $[33,36]$. Das flexible Bronchoskop dient dabei für eine Intubation als Leitschiene [28].

\section{Akutmanagement}

Die Akutversorgung beginnt wie bei jedem Trauma nach der ABC-Regel (Airway, Breathing, Circulation). Die Sicherung der Atemwege hat oberste Priorität, allerdings sollte bei einem spontan atmenden Patienten mit guter Oxygenierung die Intubation soweit als möglich vermieden werden. Bei zunehmender Luftnot und gestörter Atmung ist die Intubation am Notfallort zur Sicherung der Atemwege allerdings lebensrettend, denn durch zunehmende Schwellung, Einblutung oder Haut- und Mediastinalemphysem kann die endotracheale Intubation irgendwann unmöglich werden. Verhindert eine Kehlkopfverletzung oder Schwellung die orale Intubation, kann am Notfallort die Koniotomie oder offene Tracheotomie notwendig werden. Die Indikation zur Notfallintubation unmittelbar nach dem Trauma ist bei gestörter Atemmechanik und Hypoxie, bei ausgedehntem Trauma, zu er- 
wartender Notfalloperation, und Verschlechterung von Vitalparametern gegeben. Das Relaxans der Wahl ist in diesen Fällen Succinylcholin [35,37]. Im Schockraum sollte bei vermutetem Tracheaabriss die Intubation über ein flexibles Bronchoskop erfolgen [33]. Bei ausgedehnten maxillofazialen Begleitverletzungen kann eine Notfalltracheotomie notwendig sein, um tracheobronchiale Verletzungen nachzuweisen und die Atemwege zu sichern [29]. Es wird empfohlen, im Rahmen der Primärversorgung mit geringst möglicher Intervention die Atemwege zu sichern und den Patienten dann in eine Abteilung mit Erfahrung in der tracheobronchialen Chirurgie zu verlegen [36].

\section{Definitive Therapie}

Jede penetrierende und die meisten stumpfen Verletzungen der Atemwege mit Kontinuitätsunterbrechung erfordern ein primär operatives Vorgehen, das an die Lokalisation und das Ausmaß von Begleitverletzungen angepasst werden muss [23].

Penetrierende Verletzungen der zervikalen Trachea werden über einen kollaren Schnitt gut erreicht. Hier ist das sparsame Débridement und der Primärverschluss anzustreben. Ein protektives Tracheostoma distal der Verletzungsstelle kann bei zu erwartend längeren Behandlungsverläufen oder nicht geklärter Kehlkopffunktion vorteilhaft sein [36]. Zusätzliche Verletzungen der Thoraxspitze können eine Sternotomie erforderlich machen [33]. Die thorakale Trachea, die Hauptkarina, der rechte Hauptbronchus sowie der proximale linke Hauptbronchus werden über eine posterolaterale Thorakotomie rechts, der distale linke Hauptbronchus von links erreicht. Alle transversalen Rupturen oder Einschnitte werden in Einzelknopftechnik versorgt, longitudinale Verletzungen können fortlaufend mit monofiler resorbierbarer Naht versorgt werden $[28,38]$. Abrisse oder Einrisse mit scharfen, durchbluteten Rändern werden mit resorbierbarer Naht primär genäht. Gewebedefekte können ggf. als Eingang für eine Tracheotomie genutzt werden, die später wieder verschlossen wird [36]. Kombinierte Verletzungen von Trachea und Ösophagus sollten primär rekonstruiert werden, gefolgt von einem aggressiven Monitoring für Komplikationen. Dabei muss vor allem auf das Auftreten tracheoösophagealer Fisteln geachtet werden [36,39]. Bei allen Patienten mit traumatischen Verletzungen der großen Atemwege sollte perioperativ eine Breitspektrum-Antibiotikatherapie angewendet werden [40].

Da stark traumatisierte Abschnitte der zentralen Atemwege nach primärer Rekonstruktion zu starker ischämischer Insuffizienz oder Bildung von Narbenstenosen neigen [28], sollten die üblichen Techniken der Bronchus-Segmentresektion mit Rekonstruktion im Gesunden Anwendung finden [38,41]. U.U. muss ein Lungenlappen geopfert werden, um das Restparenchym sicher zu erhalten. Abrisse der Hauptbronchien können initial übersehen werden, da das umgebende Bindegewebe zunächst eine gewisse Schienung bietet. Innerhalb von 1-4 Wochen kommt es dann allerdings zu einem Verschluss durch Granulationsgewebe mit nachgeschalteter Lungenatelektase. In dieser Situation sind im Sinne einer sekundären Versorgung die Techniken der Bronchusresektion und Anastomosierung gefordert $[38,41]$.

\section{Interessenkonflikt: Nein}

\section{Literatur}

1 Schneider T, Volz K, Dienemann H et al. Incidence and treatment modalities of tracheobronchial injuries in Germany. Interact Cardiovasc Thorac Surg 2009; 8: 571-576

2 Gomez-Caro A, Ausin P, Moradiellos FJ et al. Role of conservative medical management of tracheobronchial injuries. J Trauma 2006; 61: 14261434

3 Palade E, Passlick B. Chirurgie traumatischer trachealer und tracheobronchialer Verletzungen. Chirurg 2011; 82: 141-147

4 Carretta A, Melloni G, Bandiera A et al. Conservative and surgical treatment of acute posttraumatic tracheobronchial injuries. World J Surg 2011; 35: 2568-2574

5 Schneider T, Storz K, Dienemann H et al. Management of Iatrogenic Tracheobronchial Injuries: A Retrospective Analysis of 29 Cases. Ann Thorac Surg 2007; 83: 1960-1964

6 Hofmann HS, Rettig G, Radke J et al. Iatrogenic ruptures of the tracheobronchial tree. Eur J Cardiothorac Surg 2002; 21: 649-652

7 Minambres E, Buron J, Ballesteros MA et al. Tracheal rupture after endotracheal intubation: a literature systematic review. Eur J Cardiothorac Surg 2009; 35: 1056-1062

8 Conti M, Pougeoise M, Wurtz A et al. Management of postintubation tracheal membrane rupture. Chest 2006; 130: 412-418

9 Chen JD, Shanmuganathan K, Mirvis SE et al. Using CT to Diagnose Tracheal Rupture. AJR 2001; 176: 1273-1280

10 Chen EH, Logman ZM, Glass PSA et al. A Case of tracheal injury after emergency endotracheal intubation: a review of the literature and causalities. Anesth Analg 2001; 93: 1270-1271

11 Cardillo G, Carbone L, Carleo F et al. Tracheal lacerations after endotracheal intubation: a proposed morphological classification to guide non-surgical treatment. Eur J Cardiothorac Surg 2010; 37: 581-587

12 Stark P. Imaging of tracheobronchial injuries. J Thorac Imaging 1995; 10: $206-219$

13 Scaglione M, Romano S, Pinto A et al. Acute tracheobronchial injuries: Impact of imaging on diagnosis and management implications. Eur J Radiol 2006; 59: 336-343

14 Orta DA, Cousar JE, Yergin BM et al. Tracheal laceration with massive subcutaneous emphysema: a rare complication of endotracheal intubation. Thorax 1979; 34: 665-669

15 Sippel M, Putensen C, Hirner A et al. Tracheal rupture after endotracheal intubation: experience with management in 13 cases. Thorac Cardiovasc Surg 2006; 54: 51-56

16 Angelillo-Mackinlay T. Transcervical repair of distal membranous tracheal laceration. Ann Thorac Surg 1995; 59: 531-532

17 Ambrogi MC, Mussi A, Ribechini A et al. Posterior wall laceration of the thoracic trachea: the transcervical-transtracheal approach. Eur J Cardiothorac Surg 2001; 19: 932-934

18 Deganello A, Sofra MC, Facciolo F et al. Tracheotomy-related posterior tracheal wall rupture, trans-tracheal repair. Acta Otorhinolaryngol Ital 2007; 27: 258-262

19 Welter $S$, Krbek T, Halder $R$ et al. A new technique for complete intraluminal repair of iatrogenic posterior tracheal lacerations. Interact Cardiovasc Thorac Surg 2011; 12: 6-9

20 Marchese R, Mercadante S, Paglino G et al. Tracheal stent to repair tracheal laceration after double-lumen intubation. Ann Thorac Surg 2012; 94: 1001-1003

21 Dienemann H, Hoffmann H. Tracheo-bronchiale Verletzungen und Fisteln. Chirurg 2001; 72: 1130-1136

22 Lee $R B$. Traumatic injury of the cervico-thoracic trachea and major bronchi. Chest Surg Clin N Am 1997; 7: 285-304

23 Huh J, Milliken JC, Chen JC. Management of tracheobronchial injuries following blunt and penetrating trauma. Am Surg 1997; 63: 896-899

24 Danić $D$, Prgomet D, Milicić $D$ et al. War injuries to the head and neck. Mil Med 1998; 163: 117-119

25 Palade E, Passlick B. Chirurgie traumatischer trachealer und tracheobronchialer Verletzungen. Chirurg 2011; 82: 141-147

26 LoCicero 3rd J, Mattox KL. Epidemiology of chest trauma. Surg Clin North Am 1989; 69: 15-19

27 Bertelsen S, Howitz P. Injuries of the trachea and bronchi. Thorax 1972; 27: 188-194

28 Symbas PN, Justicz AG, Ricketts RR. Rupture of the Airways from blunt Trauma: Treatment of complex Injuries. Ann Thorac Surg 1992; 54: 177-183

29 Bölükbas A, Ghezel-Ahmadi D, Heesen C et al. Intrathorakale Verletzungen. Chirurg 2012; 83: 91-99 
30 Kiser AC, O'Brien SM, Detterbeck FC. Blunt tracheobronchial injuries: treatment and outcomes. Ann Thorac Surg 2001; 71: 2059-2065

31 Lloyd JR, Heydinger DK, Klassen KP et al. Rupture of the main bronchi in closed chest injury. Arch Surg 1958; 77: 597-605

32 Inci I, Ozcelik C, Tacyildiz I et al. Penetrating chest injuries: unusually high incidence of high-velocity gunshot wounds in civilian practice. World J Surg 1998; 22: 438-442

33 Rossbach MM, Johnson SB, Gomez MA et al. Management of major tracheobronchial injuries: a 28-year experience. Ann Thorac Surg 1998; 65: $182-186$

34 Johnson SB. Tracheobronchial injury. Semin Thorac Cardiovasc Surg 2008; 20: 52-57

35 Rathlev NK, Modzon R, Bracken ME. Evaluation and Management of Neck Trauma. Emerg Med Clin N Am 2007; 25: 679-694

36 Farzanegan R, Alijanipour P, Akbarshahi $\mathrm{H}$ et al. Major airways trauma, management and long term results. Ann Thorac Cardiovasc Surg 2011; 17: $544-551$
37 Mayglothling J, Duane TM, Gibbs M et al. Emergency tracheal intubation immediately following traumatic injury: An Eastern Association for the Surgery of Trauma practice management guideline. J Trauma Acute Care Surg 2012; 73: S333-S340

38 Bölükbas A, Schirren J. Parenchyma sparing bronchial sleeve resections in trauma, benign and malign diseases. Thorac Cardiovasc Surg 2010; 58: $32-37$

39 Weiman DS, Walker WA, Brosnan KM et al. Combined tracheal and esophageal trauma from gunshot wounds. South Med J 1996; 89: 208-211

40 Goudy SL, Miller FB, Bumpous JM. Neck crepitance: evaluation and management of suspected upper aerodigestive tract injury. Laryngoscope 2002; 112: 791-795

41 Shrager JB. Tracheal trauma. Chest Surg Clin N Am 2003; 13: 291-304 